Décadrages Décadrages

cinéma, à travers champs Cinéma, à travers champs

11 | 2007

Terrence Malick

\title{
Mue de la nature dans Days of Heaven
}

Jean-Michel Durafour

\section{OpenEdition}

Journals

Édition électronique

URL : http://journals.openedition.org/decadrages/396

DOI : $10.4000 /$ decadrages.396

ISSN : 2297-5977

\section{Éditeur}

Association Décadrages

\section{Édition imprimée}

Date de publication : 1 octobre 2007

Pagination : $30-40$

ISBN : 978-2-9700582-6-7

ISSN : 2235-7823

Référence électronique

Jean-Michel Durafour, « Mue de la nature dans Days of Heaven », Décadrages [En ligne], 11 | 2007, mis en ligne le 01 octobre 2008, consulté le 23 avril 2019. URL : http://journals.openedition.org/ decadrages/396; DOI : 10.4000/decadrages.396

® Décadrages 


\section{Mue de la nature dans Days of Heaven}

par Jean-Michel Durafour

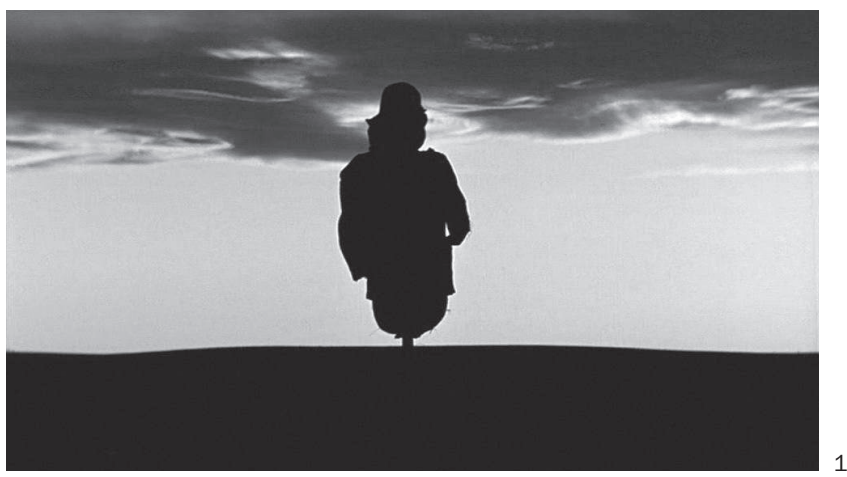

- (fig. 1): C'est à peine un homme, quand les champs, au lever du jour, ne sont pas encore quadrillés par les récolteurs saisonniers. Ce n'est déjà plus un homme, invraisemblablement arrivé en avance, ou resté en arrière, en retard, en vigie, la nuit durant: c'est un trait, une jonction entre le ciel et la terre, peut-être, avant tout, pour ces "moissons du ciel", ou, presque par anticipation, une allumette s'embrasant, sous perfusion solaire, ce plasma invasif qui n'attend pas d'être cicatrisé pour mettre le film en péril, comme, dans un instant, Bill (Richard Gere) jouera avec le feu en poussant sa fiancée dans les bras d'un fermier malade - comme, un peu après, Abby (Brooke Adams) finira par éprouver cette petite flamme pour leur "victime»- comme, plus tard encore, la jalousie du mari sera à l'origine de l'incendie nocturne du champ dans lequel, pour le moment, ce qui n'est pas un homme se tient debout. Au fait, c'est un épouvantail, immobile dans le vent, devant l'inimitable des blés crispés par l'aurore qui vient et décline, dont la venue est le déclin: on le reconnaît au bâton qui lui sort des hanches. C'est donc un homme-tige. Homme réduit à l'état de plante - ainsi que Bill, qui parodiera avec sa petite sœur un numéro de ventriloque, voudrait que le fermier, qu'un homme, le soit, en sursis, pas plus résistant qu'un pantin, manipulable à volonté, flexible, déjà mort. C'est surtout, a contrario, une excroissance 
du blé en homme, l'homme né de la végétation et retourné à elle, cet homme dont Thoreau, l'un des apologistes de la pensée transcendantaliste américaine, voulait qu'il poussât comme et avec les plantes $\mathbf{1}$ : peau, muscles et organes de chaume au milieu de plants et d'épis dont il est, dans leur dévoilement progressif hors de l'obscurité, comme le prolongement, l'annonce, le fanion, complice d'une nature dont il est censé nous protéger (les oiseaux) mais contre laquelle il ne tentera ni ne pourra rien (les criquets).

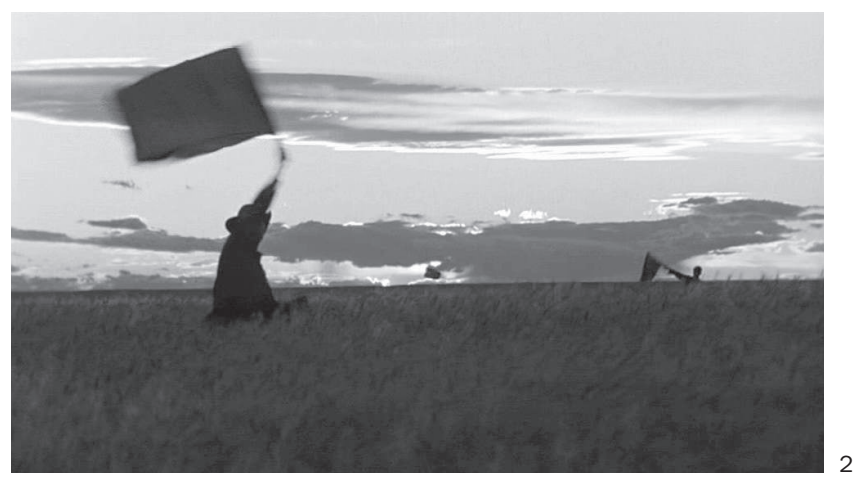

- (fig. 2): Pourtant, voilà que, dans la collure et le "contre-champ» d'un plan intermédiaire, cet épouvantail, malgré le premier plan fixe, a bougé. Il ne bouge pas: il a bougé. Il a toujours déjà bougé. C’était donc bien un homme - d'ailleurs, il n'est pas venu, il était déjà là -, de vent, de perte et de moelle, aux mains agiles dans l'air plastifié des épées de l'été. Maintenant qu'il fait jour, nous pouvons le vérifier, là où la silhouette monochrome et plate aperçue dans le contre-jour avait pu nous induire en erreur. C'était un homme, puisque les épouvantails, c'est attesté, n'ont pas de mains. Ils ont des jambes: sinon comment tiendraient-ils debout? Ils ont des yeux: ne veillent-ils pas sur les plantations? Ils ont un ventre, bien rempli d'ailleurs : ne seraient-ils pas déjà à terre sans cela? Ils ont une bouche: ne croquent-ils pas les enfants terrifiés? Mais leurs "mains» de foin, au bout de bras raides, à quoi peuvent-elles leur servir? Elles n'ont d'ailleurs rien de la fonctionnalité minimale d'une main, avec leurs doigts de brins, la gerbe paillée de leur chair feinte. Or, les mains de Sam Shepard, le fermier, sont désormais filmées en gros plan, dans leur motilité, leur souplesse, leur incarnation : caresse, empoignée, frottement. Alors que le plan d'ouverture, par sa coloration sanguine et son jeu d'ombres, sollicitait exclusivement la
1 Henry David Thoreau, Walden, édité par William Rossi, Norton \& Company Inc., New York, 1992 [1854], p. 104: "J'en arrivai à aimer mes rangs de haricots, bien plus que je ne le voulais. Ils me liaient à la terre; et je devenais fort comme Antée.". 
2 Dora Vallier, Lire Worringer, préface à Wilhelm Worringer, Abstraction et Einfühlung. Contribution à la psychologie du style, traduit par Emmanuel Martineau, Klincksieck, Paris, 2003, p. 13. Pour plus de détails sur l'haptique, on consultera Aloïs Riegl, Spätrömische Kunstindustrie, Verlag der Kaiserlich-Königlichen Hof-und Staatsdruckerei, Vienne, 1927 [1901], ainsi que Adolf von Hildebrand, Das Problem der Form in der bildenden Kunst [1893], traduit en français par Eliane Beaufils sous le titre Le Problème de la forme dans les arts plastiques, L'Harmattan, Paris, 2002. dimension optique de la vue, celui-ci s'adresse avant tout à sa propriété haptique, au sens où Aloïs Riegl l'avait définie à propos des bas-reliefs romains, analogon visuel du toucher, s'appuyant sur la "vision rapprochée» (Nacbbild) théorisée par Adolf von Hildebrand dans Das Problem der Form in der bildenden Kunst, image à "tâter du regard»2. Touche double, au demeurant: celle de notre regard sur des mains palpant des épis pour en écosser les caryopses qu'elles porteront à la bouche, dans un geste qui, à la fois, donne à voir la constitution de la figure initiale de l'épouvantail, non pas fait par l'homme, mais avec l'homme (le blé ingéré tel quel dans l'abdomen), ainsi que, à l'autre extrémité du spectre, sa vocation "céleste»: la scène suivante, celle de la bénédiction des récoltes, confère, a posteriori, à cet avalement une valeur eucharistique. L'boc est enim corpus meum d'une sorte d' "infra-substantiation" panthéiste : c'est du blé, et c'est mon corps, parce que c'est vraiment du blé. N'allez rien chercher au-delà... Dans le ventre d'un homme, il n'y a que de l'herbe. Ce n'est pas tant que ce pain est corps que le corps n'est que du pain en puissance. Thoreau, dont j’ai déjà rappelé la présence indirecte ici, voyait la terre comme un "grenier à semences": les hommes, aussi, comme le confirment de nombreux passages des Evangiles, autre texte essentiel de la culture américaine, ne sont que des graines (bonnes ou mauvaises).

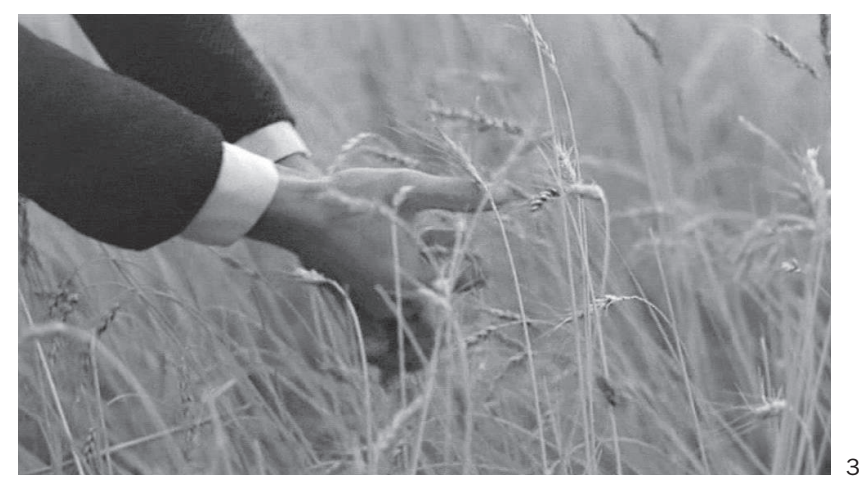

- (fig. 3): Maintenant, revenons un peu en arrière... : un plan médian sépare, l'un de l'autre, les deux précédents. Un plan où le jour gagne, et qui permet de passer de l'un à l'autre. Que montre ce plan? Une mise en mouvement, car il ne faut pas s'y tromper: ce que nous voyons, ce ne sont pas des hommes, ce sont des épouvantails en branle-bas de moisson. Le même contre-jour qu'au premier plan les habille. Aussi les épouvantails, 
récurrents dans le film, n'ont-ils rien de ce que James Morrison et Thomas Schur, dans The Films of Terrence Malick décrivent: "une grotesque imitation d'être humain (an insensate imitation of human being)»3, sans vie. Dans Days of Heaven (Les Moissons du ciel, 1978), Malick nous met en garde de ne pas méconnaître ce qui est inanimé, que là où nous pourrions avoir la tentation de voir de l'inanimé, et, le cinéma étant mouvement, de le délaisser, séjourne (déjà ou encore) une forme de vie (un homme donné pour condamné qui se maintient inexplicablement), et qu'inversement ce qui déferle sait aussi agir par densité, par "ostéogenèse", par contracture (des nuées de sauterelles tenaces capables de dévorer des milliers d'hectares en quelques heures). Le vivant est un tout; tout est vivant. Cela explique le ton quelque peu "fairy tale» du film, soutenu par la voix-off d'une enfant (Linda), qui peut seule en détenir le secret, évoluant tour à tour entre, d'une part, l'idéalisation romanesque et inventive de son séjour rural vécu principalement sur le mode ludique (rondades, parties de football...), à l'issue d'une extraction hors de la ville (une fuite après une rixe) assez proche, dans son principe, de celle de The Wizard of Oz (Le Magicien d'Oz, Victor Fleming, 1939), et, d'autre part, l'inquiétude (liée à la maturité sexuelle) de voir son frère la quitter pour une autre femme "de son âge" (comme l'atteste la première phrase), et donc de devoir sortir de l'enfance. Toute la narration est ainsi semée d'incohérences (comment Linda détient-elle des informations sur ce que ressent le fermier?), d'ellipses (voir la sécheresse explicative de la première scène d'exposition), de raccourcis (la partie citadine introductive est abrégée au plus vite). C’est que, précisément, il ne faut pas se fier à notre intellect logiciste qui classifie, compartimente, sectionne pour agir et s'orienter, et pour lequel, seul, il existe "une ligne de démarcation", une mince ligne rouge avec interdiction de franchissement entre la matière inerte et la vie, là où il n'y a, en réalité, comme l'enfant encore proche de la nature en a l'intuition, qu'un «tout indivisé» engendré par un même «flux» vital octroyant ses faveurs, non par fractures, mais par paliers imperceptibles pour notre intelligence 4 .

\section{Cinéma : méta-univers ?}

Dans une vie antérieure, Terrence Malick a été philosophe: élève de Stanley Cavell, enseignant au MIT, commentateur de Heidegger. Tout cela a souvent été rapporté. C'est d'ailleurs précisément au moment où est éditée sa traduction du Principe de raison, aux presses de la Northwestern University, en 1969, que Malick, alors récent titulaire d'un Master of Fine Arts de l'AFI Conservatory, et assurant quelques piges ici ou là, réalise son premier film, un court métrage de fin d'études, Lanton Mills.
3 James Morrison et Thomas Schur, The Films of Terrence Malick, Westport, Praeger Publishers, 2003, p. 100.

$\mathbf{4}$ Les termes entre guillemets sont empruntés à Henri Bergson, L'Evolution créatrice, PUF, Paris, 1959, dont le souhait de réconciliation entre l'inerte et le vivant est proche des préoccupations malickiennes. 
5 Qui s'inscrirait alors dans la continuité d'une école américaine de pensée représentée, outre par les transcendantalistes comme Emerson ou Whitman, et avant eux les "agrarianistes", par Mark Twain, Frederick Jackson Turner, entre autres.

6 "Combien la Nature nous rend semblables à des dieux avec si peu de choses, et si simples!" (Ralph Waldo Emerson, "Nature" dans Carl Bode et Malcom Cowley (éd.), The Portable Emerson, Penguin Books, New York, 1981, p. 14).
Fort de ce qu'un cinéaste, a fortiori américain, pouvait aussi passer pour un intellectuel, chacun y est allé de ce qu'il pouvait y avoir de "philosophique» dans un tel cinéma. On a, ainsi, fréquemment convoqué Emerson, sur fond de quelques accents rousseauistes, pour éclairer le propos malickien $\mathbf{5}$ : 1'homme ne pourrait être lui-même qu'en harmonie avec la nature, une nature d'ailleurs fortement emprunte de spiritualité 6 , là où la société le dé-nature et où l'individualisme matérialiste moderne le conduit à sa perte (dans Days of Heaven, elle fait d'un ouvrier qui ne demande qu'à "s'en sortir», qui pensera s'en sortir en sortant de la société, un hors-la-loi qu'elle rattrapera et finira par abattre après l'avoir pris en chasse comme un daim); seule la nature peut faire de l'homme un être moral. On verra alors l'ingestion du blé par le fermier comme un purgatif, un symbole de retour à la nature nourricière sur la règle "on devient ce que l'on mange». Cette idéologie "primitiviste", à rebours de tout un discours positiviste, semble, en effet, partagée par Malick: dans ses quatre longs métrages, la nature est recherchée comme un terminus ad quem dans lequel l'homme, tout en étant homo faber et se différenciant du reste du vivant par la technique (l'agriculture, par exemple), pense conquérir son être propre, et fuir la compagnie des hommes, synonyme de conformisme contraignant (Badlands / La Balade sauvage, 1973), d'autodestruction (The Thin Red Line / La Ligne rouge, 1998) ou de misère (Days of Heaven).

Cette rupture avec une compréhension anthropomorphique du monde, qui veut que l'on doive s'absorber dans le tout pour accéder à son essence personnelle (en y perdant son égotisme individuel), tel Bill, pour qui ce sera sa dernière apparition à l'écran, criblé de balles, devenu littéralement inconscient et qui sera «bu " (contre-plongée prise sous l'eau) par la rivière dans laquelle il s'effondrera, est particulièrement manifeste si l'on considère trois caractéristiques de Days of Heaven, comme d'ailleurs des autres longs métrages de Malick:

1- L'abondance des plans animaliers. La décomposition de la logique narrative, souvent brouillonne, fait que de nombreuses scènes semblent constituées de plans comme accumulés au hasard: il n'en est rien. Les plans sur les animaux - un véritable bestiaire; en vrac: antilopes, bisons, sauterelles, dindons, loutres, tigres et éléphants (sur un livre illustré), panoplie de gorille du cirque volant... - visent, au-delà d'un simple album de photos chic de type National Geographic, à déloger l'homme qui habite notre regard pour nous faire voir, par le truchement du dépassement des conditions de la perception ordinaire que le cinéma, caméra et montage, autorise (très gros plans sur des insectes, prises de vue au ras $\mathrm{du}$ sol, brusques changements d'angles), comme si nous nous tenions 
à l'intérieur de la nature, et non à partir de la transcendance de notre regard de spectateur, à la fois dans le monde et en dehors d'un monde qui se tient devant lui 7 : si les criquets sont une catastrophe pour les fermiers, ils font le régal de canards qui n'en demandaient pas tant. Pour la nature, nos petites histoires n'ont pas plus, ni moins, d'importance, dans le cours de l'univers, lequel reste impassible et continue sa routine (les grillons de chanter, les graines de germer), que la rumination d'un bovidé ou le délogement d'un putois par un visiteur importun. Entre autres exemples, le principe cinématographique classique de l'identification est largement remis en cause (le couple principal, malgré la séduction des interprètes, est relativement terne; l'époux, personnage plus attachant par son amour maladroit et sa vulnérabilité, n'a pas de nom; Linda n'est qu'une fillette esseulée). Il n'est pas jusqu'à la musique utilisée à plusieurs reprises, dont au générique d'ouverture, un extrait du Carnaval des animaux de Saint-Saëns ("Aquarium»), qui n'en relaie le motif, en faisant signe, indirectement, vers l'un des plans les plus énigmatiques du film: celui d'un verre en cristal chu au fond de la rivière et autour duquel un poisson va et vient.

2- "L'inécoute» des personnages. Michel Chion: Days of Heaven, alors que le film exploite remarquablement le nouveau système Dolby dans la restitution minutieuse des multiples murmures de la nature, et que certaines scènes sont construites entièrement sur la (perception de la) parole (ainsi la conversation hors-champ où Bill apprend l'alarmant état de santé du fermier), est "sous-verbalisé»8. Linda se contredit; de nombreux faits importants (comme la nature de la maladie de Shepard ou de la relation entre Bill et Abby) ne sont pas explicitement énoncés; les suites de la mort de Bill sont complètement passées sous silence et tout se poursuit comme s'il n'avait jamais existé ; plusieurs dialogues ne nous parviennent pas, recouverts par un fracas qui les assourdit (lors de la première scène dans la forge) ou affaiblis par la distance (quand le fermier épie les deux amants depuis son toit). Outre la référence au cinéma muet, avec la projection de The Immigrant (L'Emigrant, Charles Chaplin, 1917), qui se tresse, s'enchâsse avec le film de Malick par l'ombre, glissant sur l'écran de fortune, du bras d'un des occupants de la salle, plusieurs scènes sont organisées comme des saynètes silencieuses, à l'instar de celle du kiosque où Shepard observe, de loin, comme un spectateur dans le public, Abby et son amoureux, juchés sur une estrade, s'embrasser à travers un voile blanc, petit théâtre d'illusions qui lui fera «ouvrir les yeux", presque par l'absence de sollicitations sonores, là où jusqu'à présent il avait toujours voulu croire ce que disait sa femme lorsqu'elle protestait de son innocence sans grande conviction, malgré ce qu'il
7 Avec le cinéma, écrivait Gilles Deleuze, "La photographie, si photographie il y a, est déjà prise, déjà tirée, dans l'intérieur même des choses et pour tous les points de l'espace..." (L'Image-mouvement, Minuit, Paris, 1983, p. 89).

8 Michel Chion, La Ligne rouge, Editions de la Transparence, Chatou, 2005, p. 75. 
9 Voir, par exemple, Michel Chion, La Voix au cinéma, Cahiers du cinéma/Editions de l'Etoile, Paris, 1993, p. 20.
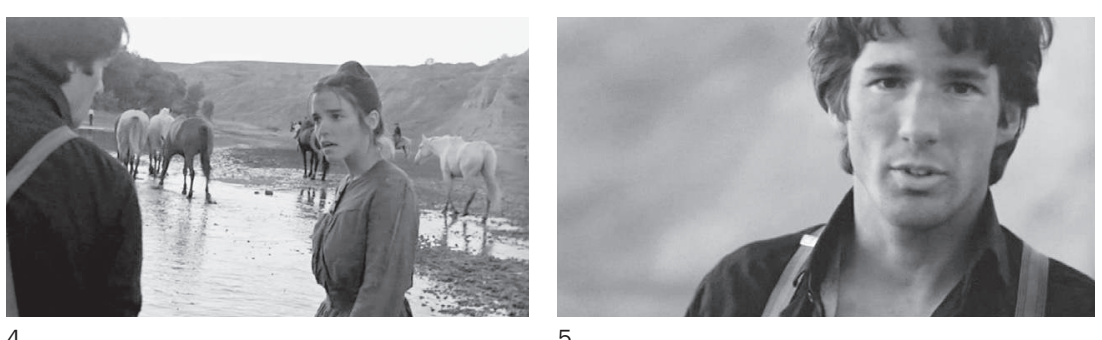

5

avait pu voir, et qui n'était déjà guère contestable. En réalité, comme on le constate, il ne s'agit pas, à proprement parler, de mutisme (du côté de l'émetteur): pas plus que dans le cinéma muet qui, comme le voulait déjà Chion, n'est pas muet mais «sourd»9. Dans un film «muet», dans les scènes "muettes" de Days of Heaven, il y a bien une parole, et une parole audible, par exemple pour les acteurs (qui ne miment pas) et l'équipe de tournage, ou, dans le récit, pour les personnages appartenant à la fiction (la scène de la fonderie) ou relativement proches de ceux qui parlent (celle du kiosque). C'est la raison pour laquelle je préfère, en filant la terminologie mise en place par Chion, parler d' "inécoute» (du côté du récepteur), pour rester dans la perspective d'une relève de la perception anthropomorphique par le cinéma, pour souligner le pouvoir du cinéma sonore que rend tangible Terrence Malick, celui de brusquement rompre l'écoute, de rendre plus éloquent ce que l'on n'entend pas parce qu'on pourrait l'entendre (dans d'autres conditions), de nous placer en deçà de notre senil d'audition habituel, de nous dérouter dans nos pratiques d'auditeurs trop souvent rendus passifs par de mauvais films ou les produits télévisuels (on tendra l'oreille; chez soi, on sera tenté de monter le son à l'aide de la télécommande...), pour nous rappeler ce que nous avons oublié, sous le prétexte que le cinéma nous fait voir: que ce n'est pas parce que le son est audible que nous devons systématiquement l'entendre, que l'audibilité n'est pas une substance, comme le voudrait un cinéma facile qui uniformise toutes les couches sonores à grands renforts de décibels, que, comme la réalité visuelle donnée à voir par le cinéma s'offre toujours depuis un point de vue, il y a, concomitamment à ces points de vue, des points d'écoute.

3-Les ruptures du montage. Dès Lanton Mills, Malick a recours aux angles larges, non seulement pour les plans sur la nature (ce qui est compréhensible), mais surtout pour les conversations (ce qui l'est moins), renonçant à l'alternance conventionnelle du champ-contrechamp et impliquant une distance de la caméra avec son objet qui nous exclut de l'intimité des propos échangés. Le point de vue retenu par Malick 


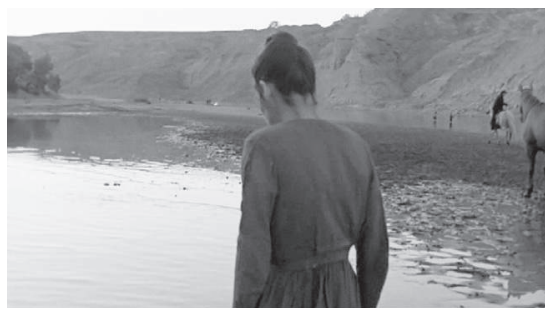

6

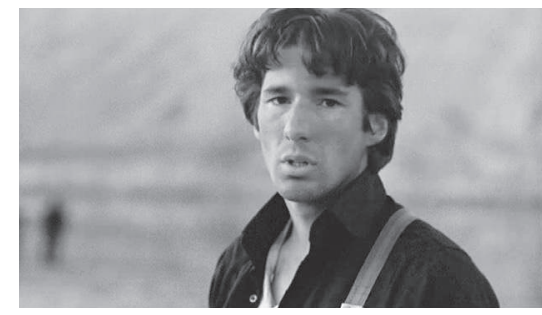

7

est ainsi, souvent, extérieur à l'un des personnages impliqués (extérieur sinon exactement à sa place physique, du moins à la place assimilable à sa position, en refusant d'introduire une partie de son corps en amorce à l'écran, par exemple), ou ne peut s'identifier à un personnage bumain potentiel de l'espace fictionnel (observant d'un peu plus loin, parmi d'autres possibilités). Cela ne veut pas nécessairement dire qu'il n'y a aucun plan "subjectif» dans Days of Heaven, mais à chaque fois ils sont vus depuis un personnage qui n'est pas en mesure de voir. J'illustrerai brièvement ce propos en citant l'exemple de deux regards de Bill à la caméra lors de la promenade avec Abby sur le gué de la rivière, quand il lui propose pour la première fois d'accepter les avances du fermier. Ils commencent par se parler face à face: Bill regarde Abby, qui le regarde (fig. 4). Aussi le plan suivant, premier regard de Bill à la caméra (fig. 5), est-il immédiatement interprété comme un plan subjectif: Abby regarde toujours Bill et la caméra vient occuper la place où elle se tient. "Malheureusement", la mise en mouvement de Bill et le travelling arrière de la caméra pour le garder au centre du cadre indiquent que les deux personnages ont repris leur déplacement. Abby également, dont le plan suivant (fig. 6) montrera qu'elle ne marche pas à reculons, mais avance en regardant devant elle, c'est-à-dire qu'elle ne peut pas être en mesure de voir Bill, qui se tient dans son dos. Nous avons ici une parfaite occurrence de ce que je désignais tout à l'heure par l'abandon de notre regard anthropocentrique et oculaire auquel le cinéma malickien nous convie. Mais la complexité de la scène ne s'arrête pas là, car ce plan, qui invalide, en termes de perception humaine, le précédent, va être à son tour invalidé par le plan postérieur. En effet, si, comme le souligne une caméra mobile portée à l'épaule, le plan sur le dos d'Abby est bien, à son tour, un plan subjectif depuis Bill apparemment localisé, il n'en est rien, car comment expliquer qu'il la voie depuis sa gauche, alors que le plan d'après, second regard à la caméra (fig. 7), semble indiquer qu'il la regarde comme s'il se tenait à sa droite? A moins de supposer que ce soit la caméra de Malick qui ait opéré une rotation d'un demi-cercle, et qui se trouve, désormais, 
non plus derrière les protagonistes, mais devant eux, sans que cela soit justifié par la progression d'un de ces personnages... Dans tous les cas, c'est l'assignation par le montage cinématographique du point de vue à un regard humainement possible qui est remise en cause par des effets de "dis-location" gigogne.

\section{La nature n'existe pas}

Mais la lecture emersonienne et "naturaliste", à l'arrière-plan de tels effets, est-elle vraiment la bonne? Non sans un peu de provocation, eu égard à la vulgate critique, je dirais que tout ce que filme Malick, c'est uniquement ceci: que la nature n'existe pas. Qu'elle est une idée toujours inventée depuis la civilisation qui nous constitue. Et c'est là, me semblet-il, que sa démarche esthétique devient la plus passionnante: dans ce constat que le cinéma, quand bien même il permet de mettre en place un nouveau paradigme perceptif (gros plan, ralenti, et ainsi de suite), est fait par des hommes, par des hommes qui rêvent qu'ils n'en sont pas, ou plus.

Au-delà du fait que le transcendantalisme emersonien est omniprésent dans la culture américaine et ne dit rien de bien idiosyncrasique sur le cinéma malickien, qui ne se distinguerait du reste du cinéma hollywoodien que par l'intensité avec laquelle il filmerait la nature, on n'a pas souvent voulu remarquer tout ce qui, dans ce cinéma, n'est pas emersonien, voire s'y oppose. Après tout, le passé agrarien que montre Malick dans Days of Heaven n'est pas si idyllique qu'on aurait pu l'attendre. La nature conduit au meurtre et à la mort: c'est sa (dure) loi. Un meurtre commis dans la confusion la plus totale, presque malgré soi ; une mort rapide, sans que la victime, traquée, hagarde, ne puisse se défendre vraiment, ni s'y préparer. Qui plus est, l'esthétisation pictorialiste des images (remarquable travail photographique de Nestor Almendros), tirant constamment l'image, volontairement, au bord du cliché "carte postale", contribue à souligner que le passé pastoral est toujours reconstruit, embelli. D’ailleurs, la "partie de campagne" sera filmée dans la même teinte sépia dominante que les inserts initiaux de photographies, empruntés à ou imitant Ansel Adams, Anne Brigman ou Jacob Riis, pour montrer que, si le film est topographiquement agencé autour de l'opposition traditionnelle entre ville et campagne, wilderness et civilisation, celles-ci ne sont, en fait, idéologiquement que les deux moments complémentaires d'un même développement historique (le train appartient à l'une et à l'autre, et les lie ensemble).

Car, si d'un côté la nature peut paraître omniprésente et référentielle dans Days of Heaven ou The Nere World (Le Nouveau Monde, 2005), d'un 
autre côté, peut-être tout aussi crucial, elle en est singulièrement absente en tant que telle, malgré les plans "panthéistes" nombreux sur la faune et la flore. Il faut revenir, pour le comprendre, à la notion de point de vue, et remonter, à rester dans la sphère philosophique, plus à Heidegger qu'à Emerson. En un sens, tout ce qui caractérisera la conception malickienne de la nature se trouve dans sa préface à la traduction du Principe de raison. A lire Malick, ce qui différencie le concept de monde (Welt) chez Heidegger, du lieu (topos) ou de la nature (phusis), c'est justement le point de vue, la manière dont ce monde apparaît à la conscience subjective, là où la pensée grecque, par exemple, envisageait plutôt la nature comme une réserve infinie de mystères. On s'étonnera donc un peu du rapprochement qu'opère Stanley Cavell entre Heidegger (Qu'appelle-t-on penser ?) et Days of Heaven, voyant dans «l'éclat formel» du film une sorte d'autorévélation de l'être de l'étant par la capacité participative des objets à leur propre représentation photographique $\mathbf{1 0}$. Une telle appréciation, si elle met l'accent sur l'influence heideggerienne, ne paraît guère compatible avec la lecture que fait Malick du philosophe allemand...

Pour l'homme malickien, la fusion emersonienne de l'ego dans la nature n'aurait donc pas vraiment de sens: la nature est toujours vue depuis une fenêtre, ou mieux depuis un cadre. Days of Heaven est structuré autour de nombreuses références picturales qui ouvrent toujours sur la nature une perspective culturelle: le célèbre House by the Railroad (1925) d'Edward Hopper, bien sûr, que la demeure du fermier reproduit quasiment à l'identique, mais aussi Christina's World (1948) d'Andrew Wyeth (fig. 8), voire plusieurs scènes de champs de Thomas Hart Benton. Voilà pourquoi tout se passe comme si la nature ne nous était jamais donnée que comme un paysage, comme (mise à) distance 11. Le paysage

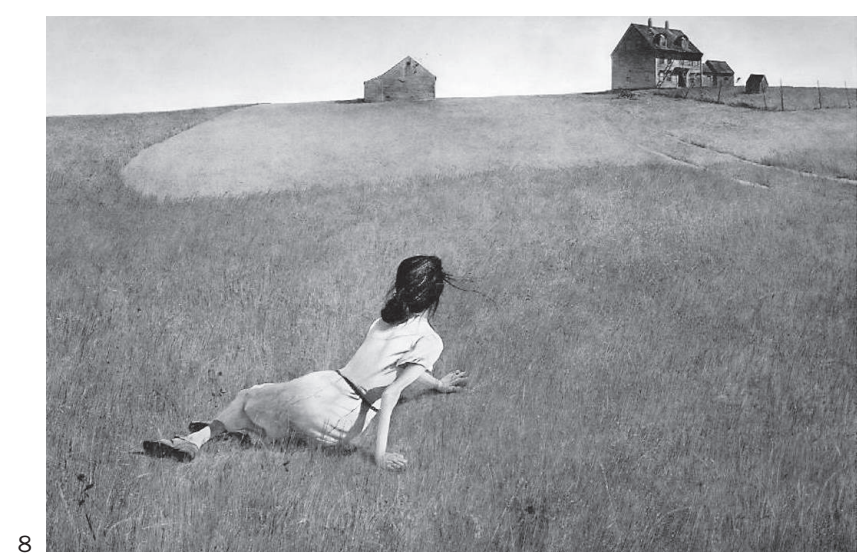

10 Stanley Cavell, La projection du monde, Belin, Paris, 1999, p. 13.

11 Anne Cauquelin rappelle d'ailleurs, dans L'Invention du paysage (PUF, Paris, 2000, p. 35), que les Grecs, pour qui la nature était si importante, n'avaient pas de mot spécifique pour désigner le paysage. 
est anti-naturel : il est paysage "de la nature», mais d'une nature qui ne s'offrirait qu'à travers le prisme d'un projet didactique du regard (artistique ou décoratif).

Il est très frappant de constater que nous, spectateurs, sommes les seuls à remarquer cette "nature» dont les personnages du film - conséquence de la dilution esthétique du regard? - semblent se désintéresser souverainement. Il ne suffit pas de l'expliquer par leur "absorbement» ou par la raison commune qu'à force d'avoir sous les yeux on ne voit plus, on ne veut plus voir, qu'il manquerait de la distance, précisément, nécessaire pour voir: c'est que, pour eux, au contraire, la nature n'existe pas, qu'elle est toujours un cadre (de vie et de travail) qui l'artificialise, qu'elle finit par devenir l'occasion d'une machination à laquelle un comploteur de palais n'aurait pas grand-chose à envier.

On voit donc ce qui compte: en mettant à mal notre regard «naturel» (par des effets de filmage et de montage dont j'ai donné quelques exemples), Malick ne le destitue pas exactement, il l'affaiblit pour le rendre sensible, comme c'est dans la maladie que nous prenons, soudainement, conscience de l'existence de nos organes. En déconstruisant notre regard, Malick veut nous faire voir ce que nous n'avons jamais vu dans l'expérience quotidienne de la vision: le relativisme (artificiel) de notre propre regard, voir non pas depuis un point de vue, mais voir le point de vue. Ce que nous voyons ne compte, dès lors, que par sa capacité (le monde déborde notre cadre de vision) à déboîter notre regard et nous en rendre présente, par le décalage, l'opération démasquée. 